\title{
BMJ Open Community-clinic linkages: qualitative provider perspectives on partnering with community health representatives in Navajo Nation
}

Christian Brown, ${ }^{1}$ Amber Lalla, ${ }^{1,2}$ Cameron Curley, ${ }^{1}$ Caroline King, ${ }^{1,3}$ Olivia Muskett, ${ }^{1}$ Shine Salt, ${ }^{1}$ Kathy Ray, ${ }^{4}$ Mae-Gilene Begay, ${ }^{5}$ Adrianne Katrina Nelson, ${ }^{1,6}$ Sonya Shin (D) ${ }^{1,7}$

To cite: Brown C, Lalla A, Curley C, et al. Communityclinic linkages: qualitative provider perspectives on partnering with community health representatives in Navajo Nation. BMJ Open 2020;10:e031794. doi:10.1136/ bmjopen-2019-031794

- Prepublication history and additional material for this paper are available online. To view these files, please visit the journal online (http://dx.doi. org/10.1136/bmjopen-2019031794).

Received 24 May 2019 Revised 24 December 2019 Accepted 17 January 2020

Check for updates

(C) Author(s) (or their employer(s)) 2020. Re-use permitted under CC BY-NC. No commercial re-use. See rights and permissions. Published by BMJ.

For numbered affiliations see end of article.

Correspondence to

Dr Sonya Shin;

sshin@bwh.harvard.edu

\section{ABSTRACT}

Objective To understand providers' opinions about the Community Outreach and Patient Empowerment (COPE) Project designed to strengthen Navajo Community Health Representative (CHR) outreach to individuals living with diabetes.

Design This was a qualitative study nested within a larger evaluation of a programme intervention.

Setting The study took place in Navajo Nation and evaluated a programme initiative designed to strengthen collaboration between CHRs and clinic-based healthcare providers and provide structured outreach to individuals living with diabetes in Navajo Nation. The CHR Programme is a formal community health worker programme that exists in most tribal healthcare systems across the USA.

Participants Healthcare providers involved in the programme took part in one-on-one interviews.

Analysis We used thematic analysis for this study. A team of three study staff used open-coding to create a codebook. Coded material were summarised and patterns were identified and tied into a narrative using concept mapping. The study design and instrument construction were guided by a Community Health Advisory Panel.

Results A total of 13 interviews were completed. Providers acknowledged CHRs as an asset to the clinical team and were enthusiastic about the COPE coaching materials, mentioning they provided a consistent message to CHRs and the community. Providers that led COPE trainings with CHRs valued the face-to-face time and opportunity to build relationships. Providers $(n=4)$ supported CHRs' access to electronic health record to record patient visits and streamline referrals. Among their requests were having designated personnel to manage referrals with $\mathrm{CHRs}$ and a formal system to record modules CHRs have completed.

Conclusion Providers participating in COPE activities valued the work of CHRs and endorsed further strengthening relationships and communication with CHRs. Healthcare programmes should consider systems changes to integrate community health workers into clinic-based teams.

Trial registration number NCT03326206; Results.
Strengths and limitations of this study

- Qualitative data present first-hand information regarding the provider experience in a communityclinic linkage programme.

- Active participation throughout the study by a Community Health Advisory Panel enriched the interview questions and interpretation of findings.

- Although saturation was obtained, the total number of participants was small and limited to providers who were involved in Community Outreach and Patient Empowerment.

\section{BACKGROUND}

In resource-poor communities, access to healthcare services is often inadequate. In these settings, community health workers (CHWs) are healthcare professionals who can link patients to clinical facilities thereby improving patient health outcomes. ${ }^{12}$ One key strength of CHWs is that they represent the communities that they serve and thus deliver outreach in a culturally appropriate manner. ${ }^{34}$

Although serving a vital role in connecting patients to the healthcare system, research has shown that CHWs have largely been structurally excluded from major aspects of the healthcare system, including clear roles and workflows within care teams, participation in team meetings and access to electronic health records (EHRs). ${ }^{56}$ Conversely, when CHWs are integrated into the healthcare system, they are effective at improving healthcare outcomes for patients. ${ }^{6-8}$ Effective integration of CHWs into the care team allows CHWs to share vital perspectives of the home environment to clinic-based healthcare providers who may have little or no insight into patient's living situation and psychosocial surroundings. By expanding communityclinic linkages and connecting CHWs with 
the healthcare system, CHWs are better able to support both the patients and providers in forming an effective patient-centred care team. ${ }^{69}$

In the USA, community health representatives (CHRs) are a long-standing workforce of community health workers who provide culturally sensitive outreach to native families living in vastly rural communities. ${ }^{10}$ Because CHRs are typically operated as tribal programmes and healthcare services are often delivered through the federal Indian Health Services, coordination between CHRs and clinical providers may be limited. In an effort to integrate CHRs with local clinic-based teams, a collaboration among the Navajo CHR Program, Navajo Area Indian Health Services (NAIHS) and Brigham and Women's Hospital was established in 2009. The goal of this initiative, called Community Outreach and Patient Empowerment or COPE, was to integrate CHRs with healthcare teams.

Evaluation efforts have sought to understand how COPE impacts clinical and health systems outcomes, as well as diverse stakeholder perspectives including CHRs, clinic-based providers and patients themselves. The impact on CHR-provider communication and clinical outcomes has been described elsewhere. ${ }^{11}$ Nonetheless, sustainable improvements in health system performance rely on a shared sense of the systems to be improved, deeply engaged staff and ongoing feedback systems. ${ }^{12}$ To inform ongoing programme improvement and understand potential for this programme as a lasting and integrated component of the local healthcare system, we sought to understand the programme's impact on the experience of clinic-based healthcare providers. To our knowledge, this is the first study to explore the providerreported perspective and recommendations towards the utilisation and integration of CHWs, including CHRs, into 'cross-institutional' healthcare teams. ${ }^{13}$

\section{METHODS}

\section{Study setting}

The Navajo Nation is a federally recognised American Indian tribe in the USA covering regions of Arizona, New Mexico and Utah. Similar to other communities with few economic resources within the USA, the Navajo Nation lacks the infrastructure to allow for consistent access to quality healthcare. This largely stems from rural nature of the reservation, which has unpaved roads, long distances from patients' homes to healthcare centres and a high degree of provider turnover within the healthcare facilities. $^{1415}$

Partially due to these inequities, diabetes and cardiovascular disease stand as some of the leading causes of death within American Indian and Alaskan Native communities within the USA. ${ }^{16}{ }^{17}$ It has been estimated that roughly 25000 Navajos have diabetes (around 21.5\% of the adult population) and another 75000 have been diagnosed with prediabetes. ${ }^{18}$ Among Navajo people, cardiovascular disease and diabetes represent the third and fourth leading causes of death, respectively. ${ }^{19}$
The Navajo Nation is divided up in to separate local governances called 'chapters'. These chapters are grouped geographically and represented by eight service units within the NAIHS. Each service unit has a health facility, sometimes with several satellite clinics.

Within the Navajo Department of Health, the Navajo CHR Outreach Program employs approximately 141 staff, including 99 CHRs. ${ }^{20}$ Navajo CHRs are required to be trained as a Certified Nursing Assistant (CNA) and must speak Navajo. Many CHRs have additionally obtained certification and credentialing beyond the CNA required for the job, including CHW certification through the state of New Mexico. ${ }^{21}$ Typical services provided by Navajo CHRs include home visits to monitor vital signs and provide health education, referrals for additional services and resources needed by their client, community outreach for urgent or emergent issues, and health fairs and other health promotion activities. Each CHR is assigned to one or two chapters (usually where they themselves live). A CHR office is usually provided by the community within the chapter house. CHR teams are organised by service units, with a team office typically located close to the service unit's main healthcare facility.

\section{COPE intervention}

The COPE intervention consists of three main strategies: creating systems for referral, coordination and communication between CHRs and clinic-based providers; providing high-quality training to CHRs on health topics and motivational interviewing; and developing standardised outreach materials for CHRs to use with patients living with diabetes. ${ }^{4}$ This intervention was delivered under programme auspices; thus, the research to evaluate COPE's impact was observational in nature.

The partnership began when staff from the Division of Global Health Equity at the Brigham and Women's Hospital were invited to collaborate with NAIHS and the Navajo Nation CHR, specifically to bring technical assistance and tools developed from international programmes in which CHWs have been successfully integrated into large-scale public health systems. ${ }^{22}$ Initially, COPE project staff met with the Navajo CHRs to determine what additional tools COPE could provide to increase the level of patient care for the CHRs. CHRs stated that a standardised set of patient teaching materials would be the most important aspect to improve their healthcare delivery on the Navajo Nation. COPE staff worked with CHRs and healthcare providers to develop culturally specific health education materials in the form of flipcharts.

COPE staff then facilitated monthly trainings on these health education materials at all eight service units. For each training, the COPE team invites a local healthcare provider to deliver the training on their specialty or area of interest and provides the training materials (learning objectives, materials and competency assessment) to the trainer in advance. CHRs complete precompetency and postcompetency assessments, and both CHRs and trainers complete a feedback form to evaluate their satisfaction with 
the event and seek further suggestions for improvement. CHRs who receive a passing score $(\geq 70 \%)$ on their postcompetency assessment are then given the flipchart to use with their patients; if they do not pass, COPE staff schedule a follow-up one-on-one training to review materials and readminister the competency assessment.

Other concerted efforts of the COPE Program included initiating case management meetings between CHRs and providers and establishing access for the CHRs in the EHR used by most Indian Health Service facilities. Nationally, CHWs have faced barriers documenting their patient encounters and communicating their observations to clinic providers. When COPE began, CHRs did not have access to the EHR. As a result of the COPE Program and supportive clinic providers, two of the service units were able to grant CHRs access to EHR. CHRs in those service units underwent Health Insurance Portability and Accountability Act (HIPAA) and information security training prior to gaining access to the electronic systems. Specific templates were created for providers to make referrals to CHRs and for CHRs to document their home visits. Improvements in communication were shown as a result of this system change. $^{21}$

\section{Study population}

Providers were included in this study if they had previous experience or involvement with the COPE Program. Roles of COPE involvement by providers varied greatly from leading CHR training, facilitating meetings between clinic staff and CHRs and referring patients to the CHR programme.

\section{Study design}

This was a qualitative study embedded within a broader observational study designed to evaluate the impact of the COPE intervention. COPE research staff approached healthcare providers via phone, email or in person to ask if they would be willing to participate in the study. For those providers who agreed to be interviewed, one of three trained study staff conducted the in-depth interviews lasting 15-35 min (CB, AL and CK). None of the providers declined interviews. Interviews were conducted in English, either in person in clinic or by phone. They were transcribed verbatim by a note taker or digitally recorded and then subsequently transcribed. Transcripts were not reviewed by interviewers. Sampling ended when saturation was achieved. The study team determined that saturation was achieved when no new information about the impact of the COPE Program on provider care surfaced after three consecutive interviews. Specifically, after 10 interviews, our team observed that three additional interviews did not contribute new information, resulting in 13 interviews total.

\section{Research team}

The research team consists of one non-Navajo, female physician who is also the study's principal investigator ( $\mathrm{ShS}$, $\mathrm{MD}, \mathrm{MPH}$ degree); five young male and female researchers working as either interns or study coordinators $(\mathrm{CB}, \mathrm{AL}, \mathrm{CK}$, $\mathrm{CC}$ and SoS), all with BA's and three of whom are Navajo (CB, CC and SoS); one Navajo, female community outreach manager at COPE who has also worked as a CHR (OM); one non-Navajo, female clinical applications coordinator at Navajo Area Indian Health Services (KR, CNM degree); the Navajo female programme director of the Navajo CHR \& Outreach Program (M-GB, MSW degree); and one nonNavajo female qualitative researcher/ research manager employed at Brigham and Women's Hospital and stationed at COPE (AKN, MPH, Msc degree). ShS has worked for 20 years as a researcher dedicated to community health interventions for patients with tuberculosis and HIV at Partners In Health sites including Haiti, Peru and Russia and has led COPE since 2009. AKN has been working with Partners In Health as a research assistant and qualtiative researcher for 9 years. M-GB has been the programme director for the Navajo CHR/Outreach Program for 18 years.

\section{Patient and public involvement}

The study was carried out using Community Based Participatory Methods, with ongoing input from a Community Health Advisory Panel (CHAP) composed of patients and CHRs. Prior to the study initiation, the CHAP provided feedback to define the overall study objectives and specifically endorse this qualitative study of provider perspectives. Study staff developed the qualitative interview guide, with feedback from the CHAP. The CHAP was involved throughout the study, during quarterly meetings in which the study team presented aspects of the study (eg, interview guide, sampling plan, preliminary findings and manuscript draft) and sought feedback through facilitated small group sessions. As an example, the provider interview guide was designed by study staff to understand the provider's experience working with CHRs and also specifically interacting with the COPE intervention. The interview guide was then presented and reviewed by CHAP, which is composed of Navajo CHRs, patients and the family members of patients. The goal of this review was to ensure the research and interview guide was culturally informed and would elicit information of interest to patients and family members. On review, the CHAP recommended adding two questions that focused on food access and traditional/holistic medicine. The final interview guide is included in online (supplementary file). CHAP also provided feedback on the study findings to ensure that interpretation of findings were consistent with their own perspectives as CHRs and patients. The public was also involved in the study by obtaining initial approval from Tribal Agency Councils and Health Boards and by formally presenting results to these same groups at the end of the study.

\section{Data analyses}

Thematic analysis was used to analyse qualitative data, in order to respond to themes emerging from the providers themselves. ${ }^{23}$ All transcriptions were deidentified and uploaded into the Dedoose software. ${ }^{24}$ Pseudonyms were assigned to each respondent. Themes were not determined in advance, rather they emerged from 
the interview data. A team of a qualitative researcher (AKN) and two research assistants (CB and AL) coded interviews using open coding. First, codes were identified by the study team, and then clustered based on team discussion into broader themes. Four themes emerged that made up the final codebook: general interaction with COPE, implementation of COPE within the service unit, the impact of COPE and improvement of COPE and related activities. Interviews were then reviewed again by the same team and coded independently. Inter-rater reliability was tested, and kappa scores of 0.49, 0.49 and 0.46 were achieved. Discrepancies in coding were discussed with a qualitative researcher and finalised based on group discussion. A brief synopsis for each code was then generated, describing the number of respondents endorsing each code, as well as patterns of concordance and contrasts among respondents. These findings were then unified into a narrative using concept mapping. When organised by theme, the narrative was somewhat redundant; therefore, the team chose to organise the narrative into three cross-cutting topics, which emerged as the most salient programme features based on provider responses. To further assess validity of coded results, findings were triangulated with field observation among COPE staff as well as CHAP feedback.

\section{Choice of terminology}

In this study, the study team decided to use the term CHR instead of CHW, which is a broader term inclusive of CHRs. Navajo CHRs refer to the community members that they work with as clients rather than patients; for this reason, we use the term 'clients' although providers may refer to the same individuals as 'patients'.

The term providers used throughout this paper is intended to be encompassing of multiple different hospital staff that have direct contact with patients. Examples of providers interviewed range from doctors, nurses, public health nurses and CHR supervisors.

The term community-clinic linkages refers to relationships between clinical provider teams and communitybased resources to improve delivery of care and health outcomes through greater communication and collaboration across three key stakeholders-the clinic/clinician, the patient and the community resource. ${ }^{2526}$

\section{RESULTS}

A total of 13 providers from five service units were interviewed (table 1). Five interviews took place by phone and eight in-person at the interviewee's worksite. Findings related to three major aspects of the programme: acknowledging the importance of community-clinic linkages, endorsement of COPE training and materials and an appreciation for CHR access to EHRs.

Acknowledging the importance of community-clinic linkages Overall, most providers (10) explicitly acknowledged the importance of the CHR role and described CHRs as

\begin{tabular}{ll}
\hline Table 1 & Occupation and number of participants \\
\hline Job title & Number of participants \\
\hline Physician & 5 \\
Diabetes educator/specialist & 4 \\
Case manager & 1 \\
Registered nurse & 5 \\
Public health nurse & 3 \\
Nurse practitioner & 1 \\
Nurse midwife & 1 \\
CHR supervisor & 1 \\
\hline
\end{tabular}

*Note: some participants have more than one title. Quotes are not linked with specific service units or job titles in order to protect participants' confidentiality.

$\mathrm{CHR}$, community health representative.

a 'big interface to allow the community to interact with the hospitals'. Providers acknowledged the unique value of CHRs' outreach work, knowledge in health education and management, knowledge of community/resources and ability to see patients in their own living environment. Through COPE interactions, providers gained a deeper appreciation of the role of CHR.

[Due to COPE], there is a closer interaction that we have with the CHRs because we both serve the same population of people. They're able to do the outreach piece of it, more so than we are. Yes, a closer interaction with the CHRs to help bridge the gap where we can't meet the patient out in the communities per se. Catherine, nurse practitioner

We were pounding home [to the CHRs]: 'You're the ones who have eyes and ears on these people, you're very valuable.' And over a period of time, they started understanding that they were an asset, that they had things to offer that no doctor, very, very few nurses, would understand, because they were in the home. They can see things that we would never see. Kate, diabetes educator

Nonetheless, almost all providers felt that there was a need for increased formalised collaboration, specifically in the form of an interdisciplinary team. Multiple providers suggested increased face-to-face collaboration through the creation of formalised multidisciplinary health teams, regular interprofessional meetings consisting of public health nurses (PHNs), CHRs, clinicians, nurses and diabetes educators. 'Arranging actual meetings, physical meetings' to learn about respective programmes could also increase an understanding of how programmes could work together. One provider expressed concern that CHRs are hard to get to know since they are not based at the hospital.

It'd be great if we could get to know certain CHRs better, so we could really maintain communication with them, and they would feel more comfortable talking 
to us... Just being able to get to know the CHRs better, I think would increase communication, and have them be more comfortable talking to me, calling me about patients, that would really change up some of the ways that we practice. Andrew, physician

[It] would be great for them to meet with the diabetes educators... Some of the requests for teaching the CHRs, it'd probably great for the diabetes educators to help because they're RN-level providers, so they could actually help with a lot of the diabetes education with the CHRs... [There are] a lot of ways that we could all kind of work together better. Janet, physician

Providers cited case management as a formalised way that many service units currently arrange for different fields to come together to discuss patient care. While more than half of the providers reported ongoing case management teams in their service unit, they described mixed feelings towards case management. While some providers (3/6 who had case management at their facility) felt that case management was useful and worth the time investment, others (2) expressed scepticism because they felt it was unnecessary or time-consuming. Providers indicated that barriers to successful case management programmes included high provider turnover, short staffing and lack of time or inflexibility in schedules.

They had never used case managers here in the diabetes program before.... So I brought the concept of case management to the table, and our CHRs began to identify some of their problem clients in the community. And we would have complex care case management meetings with the public health nurse, the doctor, all the family members, and tried to get as much clear understanding of what some of the problems or barriers to care were. And, before long the CHRs and the public health nurses began working really, really, really close together. We were just seeing the success of the whole idea, the whole program. Kate, diabetes educator

[Would case management be useful?] It just depends on the patient and how much time people have to do meetings. I know PHN do, but I usually don't have time. For me it's better if I do individual follow up on someone I've referred. Rosita, diabetes educator

Lastly, many providers mentioned their own or their colleagues' lack of awareness of the COPE Project. Because of high provider turnover rates, one provider suggested quarterly meetings with progress updates as 'an opportunity to educate the people that have just joined the staff'. Providers suggested that increasing awareness and sharing results about COPE could increase referrals and reach more beneficiaries.

As a clinician, I want to know outcomes. If someone is going to take the CHRs' time and resources, to send them to a place to do this, I want to know that it works, you know? Abigail, physician

If we were more aware of [CHRs], we probably could reach out to them more and utilize them more and they could utilize us more too. Janet, physician

If... we could collaborate more it would benefit the patient population that we work with. There's many people that could be seen, and I think that sometimes, because of the overwhelming numbers, not as many patients get touched because we don't even think about referring. Tonya, diabetes specialist

\section{Endorsement of COPE training and materials}

Eight of the providers felt that the health education materials and trainings provided by the COPE Program helped to foster collaboration with CHRs by increasing message consistency. Most providers recognised the 'COPE Flipcharts' as a powerful health education resource for CHRs, patients and the clinics. Providers reported the large variety of topics and use of visuals in the COPE health education materials were thought to be helpful in relaying information.

There's a lot of health topics that they've covered. When I see their monthly [training] activity, there's a lot of topics, using the flipchart and having a resource available that they can use. Rosita, diabetes educator

Making the modules, the little flipcharts, I think that's a good idea for staff to have. They pull it out for a topic, and I know it's consistent with what we teach.... and I know that they're competent to teach what they're teaching. That makes a big difference. Harriett, CHR supervisor

Interestingly, by increasing the consistency of the information taught by both clinical and homecare teams, the health education materials also built trust and confidence in the CHRs among providers and patients. Many providers felt that having provider-led trainings aided in delivering a consistent message to CHRs and ultimately to the patients.

Through bolstering the education that the community health workers receive, I think it's making sure what their story is matched by what the patients receive in the hospital as well. We've always had a disconnect, historically... In other words, the community health worker might tell the patient one thing, and then the patients receives completely different information in the hospital, and now the patient doesn't trust anybody. And now,... the patient has trust because they're hearing the same information because the community health worker is now educated, and COPE has been that bridge. Charlene, nurse midwife

Having the providers, the primary care providers, participate in education with the CHRs, I think, is really important because that way the providers are more likely to refer to the program because the providers 
have the confidence... about the types of information the CHRs are going to provide and their knowledge base. Janet, physician

Providers felt that educational materials could be further used to reach a broader audience. While providers reported feeling that both the educational materials and flipcharts were helpful, some providers felt that the materials were currently underused and that it would be helpful to record which CHRs had received training in which modules. One provider noted the challenges around discussing difficult topics, such as alcohol use. This provider suggested the 'COPE Flipcharts' could provide CHRs with more confidence in addressing these topics and patients could be more receptive.

The education is something that works well. I do think that more patients could benefit from the education that COPE is providing. Tonya, diabetes specialist

The majority $(n=8)$ of providers interviewed indicated they had delivered at least one CHR training using COPE materials. Providers stated that the trainings ultimately increased face-to-face time between providers and CHRs. During these interactions, the providers felt they were also able to directly address CHR concerns and questions.

It was nice that COPE has started to invite the providers to give some of those teaching sessions, because that way we can make sure the message the CHRs are delivering is in line with the same message that like we deliver to patients when they come to their clinic visit. Janet, physician

My one interaction was them face to face has been with the talk I gave. I was able to address some concerns that they had. Gerry, public health nurse

Providers indicated, however, a lack of clarity around which CHRs were trained in which COPE modules (topics) and how they were using the flipcharts in the homes. Providers stated that knowing what topics the CHRs were trained to deliver could help them identify the best patients for referral and request specific health education topics for individual patients. One provider suggested possible regular updates on recent CHR trainings.

I think they have all the materials, kind of like resources, but I don't know how [they] actually use them. I don't actually know if they go through the whole curriculum... Charlene, nurse midwife

\section{CHRs' access to EHRs}

Ten of the providers supported systems to improve communication with the CHR team, and four specifically endorsed CHR integration into EHR to record patient visits and streamline referrals, regardless of whether their service unit currently had this system in place.

Two providers from a service unit where CHRs had access to EHR reported they were aware that CHRs in their service unit had access to the EHR. These providers stated that their ability to send referrals to CHRs through the EHR has been valuable in seeing CHR notes with ease. Providers also felt the EHR helped to close the feedback loop between their practice in the clinic and the CHR's combined community outreach and public health education. They then acknowledged that this increased the ability for CHR-provider interaction and enabled providers and CHRs to collaborate more easily. Providers reported they used the EHR to share information about their patients with the CHRs. In some cases, providers reported that they would modify their care plan based on critical data provided in the CHR notes. Overall, providers reported that the feedback from the CHR through the EHR system was both useful and appreciated.

When I see the patient and I kind of look through their chart, and I see that the CHRs left a note, I will say, 'I hear that one of our CHRs came out to visit you, they were mentioning this that and the other, I'm concerned about that.'... and then sometimes, if that means that I need to see the patient sooner, I might try to schedule the patient sooner. Andrew, physician, site with CHR access to EHR

In contrast, providers who worked in service units without access to EHR reported difficulty in locating CHR documentation and receiving feedback from their referrals to the CHRs. Providers stated that they felt CHR's paper records from their home visits were cumbersome to read and providers eventually stopped requesting them. They also described frustration when they failed to hear back from the CHR after making a referral due to limited follow-up communication. They felt that they could not determine whether the patient was successfully visited or enrolled in the CHR's care nor discern what occurred at these visits.

There are challenges because we don't share EHR electronic notes. So we don't always get a chance to see what is being done with that CHR and patient in the community. I mean they do a note, and it probably goes in the [paper] chart, but ... if it's not in EHR, I really don't pull a chart unless I absolutely have to. Catherine, nurse practitioner, no EHR access

When I put in a referral, I don't get notified.... Sometimes I don't get any feedback in terms of what was the outcome of the [CHR] visit, [or] if there was any interaction done at all with some of the patients. Gerry, public health nurse, no CHR access to EHR

Providers at sites where CHRs did not have access to the EHR endorsed the prospect of granting CHRs access to EHR in the future. One provider stated that receiving feedback from CHRs via EHR would encourage providers to continue referring patients to the CHRs because they would be able to see the results of their referral and further incorporate the CHR notes into their patient's treatment plan. 
Getting those EHR notifications, I think it is really helpful because if you refer to a program but you never get any feedback on how your patients are doing, or what's happening, it's kind of discouraging and people often won't continue to refer if that's the case. So, I think like getting some sort of feedback about what interventions are performed with the patient, how their doing, things like that really encourage the providers to continue to refer. Janet, physician, service unit with no EHR access

\section{DISCUSSION}

Three main themes emerged from the perspectives of the providers: an acknowledgement of the importance in developing clinic-community linkages, an endorsement of the COPE materials being delivered by the CHRs and the necessity for CHRs to be integrated into the EHR. The themes that emerged are similar to other situations on a national level where healthcare providers grapple with a further integration of CHWs into clinical care teams. ${ }^{97}$ Although difficult, other studies have shown positive outcomes from this integration. ${ }^{28}$ The remarks from providers in this qualitative study works to support the current literature on this subject and encourages integration of the 'quadruple aim'-that of improving provider satisfaction-alongside the standard triple aim of enhancing patient experience, improving population health and reducing costs. ${ }^{29}$ One recommendation to address provider satisfaction is creating healthy 'care teams' including expanded roles that allow delivery of preventive care and health coaching to patients. The importance of achieving a sense of joy and self-efficacy among healthcare providers highlights the challenges of staff burnout, particularly in settings where staff turnover and workload is high.

Providers recognised the critical role of the CHRs in the healthcare team and felt that increased interactions (eg, informational exchanges, interprofessional meetings including case management and quarterly progress updates) would strengthen the relationship between CHRs in the field and providers in the clinic.

Almost all the providers interviewed responded positively to the COPE materials and the quality of the health education that CHRs were delivering in their communities. By involving local providers in the development of educational materials and delivery of training, providers had confidence in the materials and felt reassured that information provided by CHRs would be consistent with what patients were being told in clinic. We felt that this was one of the strongest aspects of the intervention. Provider-led trainings increased collaboration with CHRs by building trusting relationships and stimulating greater collaboration.

Our findings support the need to integrate CHRs through shared access to the EHR. EHR access has provided a quick and streamlined process to refer patients to CHRs and critical sharing of important information that improved providers' ability to care for their patients. As healthcare systems move increasingly towards paperless systems, providers rarely review hand-written notes by CHRs. ${ }^{30}$ This provider perspective highlights the importance of advocacy among clinicians to facilitate CHR access to EHR. Across tribal communities, many Indian Health Service sites use the same EHR system, providing a unique opportunity to use training and clearance protocols as well as EHR templates developed in Navajo to any interested site.

Our study has several limitations. Because COPE team members conducted the interviews, providers may not have been as truthful about negative experiences and interviews may have been less comfortable probing for negative feedback. However, the interview guide included questions explicitly asking about implementation challenges and opportunities for improvement. We feel that providers were honest about their experiences with the COPE Program, the Navajo Area IHS System and the Navajo Nation CHR Program based on their wide range of responses including frank discussion of challenges in the programme. All of the providers that were interviewed as a part of this study had some relationship to the COPE Program and were therefore more likely to be advocates for the programme and for CHRs in general. We recognised that these responses do not necessarily reflect perspectives among all providers across these healthcare facilities. While not the scope of this study, interviewing providers not involved with COPE could have provided a more accurate reflection of the general population of providers, as well as insight on how to involve more providers. In fact, those who participated in this study felt that more providers should be aware of this programme and the role of CHRs. They suggested that the materials could be used to provide health coaching to more patients and also emphasised the need to increase awareness of CHRs and the COPE intervention among the broader community of clinic-based providers.

\section{CONCLUSION}

Providers who worked with CHRs and the COPE intervention expressed a strong appreciation for the unique role of CHRs and the value of COPE's standardised health education materials and CHR trainings. At a system level, providers were able to work more closely with CHRs when they shared access to EHRs. Successful integration of CHWs into interprofessional healthcare teams may inform CHW programmes across a variety of settings. Further research to better understand the patient experience would complement the provider perspective to determine whether and how integration of CHWs into healthcare teams also improves the patient experience of care.

\section{Author affiliations}

${ }^{1}$ Division of Global Health Equity, Brigham and Women's Hospital, Boston, Massachusetts, USA

${ }^{2}$ University of New Mexico School of Medicine, Albuquerque, New Mexico, USA ${ }^{3}$ School of Medicine, Oregon Health \& Science University, Portland, Oregon, USA ${ }^{4}$ Navajo Area Indian Health Service, Saint Michael, Arizona, USA

${ }^{5}$ Community Health Representative Outreach Program, Navajo Nation Department of Health, Window Rock, Arizona, USA 
${ }^{6}$ School of Public Health and Tropical Medicine, Tulane University, New Orleans, Louisiana, USA

${ }^{7}$ Department of Global Health and Social Medicine, Harvard Medical School, Boston, MA, United States

Acknowledgements We wish to thank the Community Health Advisory Panel members for their thoughtful contributions to this study.

Contributors CB led the qualitative analysis team as a junior investigator. He coded interviews, conducted analysis and drafted all sections of the manuscript. AL was part of the analysis team and helped conduct and code interviews, conduct analysis and edit the final manuscript. CC was part of the analysis team and helped to code interviews, conduct analysis and edit the final manuscript. CK conducted and transcribed interviews and provided edits on the final manuscript. OM was part of the analysis team and provided input on the results and final manuscript. She is a Navajo researcher with experience working as a CHR. ShS was part of the analysis team and helped to code interviews, conduct analysis and edit the final manuscript. KR works for Indian Health Service and is involved in the design, maintenance and advocacy for the use of the electronic health record (EHR) across all Navajo health sites. She provided feedback about the EHR and its function within the Navajo health facilities during data collection and analysis and edited the final manuscript. M-GB is the programme director of the Navajo Nation Community Health Representative and Outreach Program. She provided feedback during the design of the study and data analysis and edited the final manuscript. AKN is a qualitative researcher. She guided the team in the methods of designing the study and conducting data analysis and incorporated edits from all authors. She provided a qualitative methods perspective to the final manuscript. SoS is the principal investigator for this study. She guided the team in the design of this qualitative study and provided high-level support in the analysis and drafting of the manuscript. She provided clinical perspective on the findings.

Funding Research reported in this publication was funded through a PatientCentered Outcomes Research Institute (PCORI) Award (AD1304-6566). The Navajo Nation Human Research Review Board as well as the Partners Institutional Review Board reviewed and approved the study protocol. All results have been presented to the Navajo Nation Human Research Review Board (Protocol \#NNR-11.150).

Competing interests ShS discloses that she has served as the executive director for a 501(c)3 organisation, entitled Community Outreach and Patient Empowerment Program, which continues to support the described programme, since study completion

\section{Patient consent for publication Obtained.}

Ethics approval The Ethics Committee of Partners Healthcare and the Navajo Nation Human Research Review Board (NHRRB) approved the study. All participants signed a consent to participate in this study.

Provenance and peer review Not commissioned; externally peer reviewed.

Data availability statement Data are available on reasonable request. All research data are property of the Navajo Nation as per NHRRB protocols. Investigators who seek to use these data would need to request permission from the NHRRB and provide reassurance that their request is consistent with applicable privacy, confidentiality and other legal requirements.

Open access This is an open access article distributed in accordance with the Creative Commons Attribution Non Commercial (CC BY-NC 4.0) license, which permits others to distribute, remix, adapt, build upon this work non-commercially, and license their derivative works on different terms, provided the original work is properly cited, appropriate credit is given, any changes made indicated, and the use is non-commercial. See: http://creativecommons.org/licenses/by-nc/4.0/.

ORCID iD

Sonya Shin http://orcid.org/0000-0003-3466-9055

\section{REFERENCES}

1 Brown HS, Wilson KJ, Pagán JA, et al. Cost-Effectiveness analysis of a community health worker intervention for low-income Hispanic adults with diabetes. Prev Chronic Dis 2012;9.

2 Condo J, Mugeni C, Naughton B, et al. Rwanda's evolving community health worker system: a qualitative assessment of client and provider perspectives. Hum Resour Health 2014;12.

3 Pérez LM, Martinez J. Community health workers: social justice and policy Advocates for community health and well-being. Am J Public Health 2008;98:11-14.
4 Gampa V, Smith C, Muskett O, et al. Cultural elements underlying the community health representative - client relationship on Navajo nation. BMC Health Serv Res 2017;17.

5 Kangovi S, Grande D, Trinh-Shevrin C. From rhetoric to reality-community health workers in post-reform U.S. health care. $N$ Engl J Med 2015;372:2277-9.

6 Allen CG, Escoffery C, Satsangi A, et al. Strategies to Improve the Integration of Community Health Workers Into Health Care Teams: "A Little Fish in a Big Pond". Prev Chronic Dis 2015;12.

7 Shah M, Kaselitz E, Heisler M. The role of community health workers in diabetes: update on current literature. Curr Diab Rep 2013;13:163-71.

8 Malcarney M-B, Pittman P, Quigley L, Horton K, et al. The changing roles of community health workers. Health Serv Res 2017:52:360-82.

9 Findley SMS, Hicks A, Chang J, et al. Community health worker integration into the health care team accomplishes the triple aim in a patient-centered medical home: a Bronx tale. J Ambul Care Manage 2014;37:82-91.

10 Navajo community health representative. Available: http://www. nndoh.org/chr.htm

11 Trevisi L, Orav J, Atwood S, et al. Integrating community health representatives with health care systems: the impact on clinical outcomes in Navajo nation. American Public Health Association Annual Meeting, Atlanta, GE, 2017.

12 Getting started kit: rapid response teams. Available: www.ihi.org

13 Sequist TD, Taveras EM, Elsie M. Clinic-community linkages for highvalue care. N Engl J Med 2014;371:2148-50.

14 English KC, Wallerstein N, Chino M, et al. Intermediate outcomes of a tribal community public health infrastructure assessment. Ethn Dis 2004;14:S61-9.

$15 \mathrm{Kim}$ C. Recruitment and retention in the Navajo area Indian health service. West J Med 2000;173:240-3.

16 American Indian and Alaska native heart disease and stroke fact sheet. Available: https://www.cdc.gov/dhdsp/data_statistics/fact sheets/fs_aian.html

17 Native Americans with diabetes. Available: https://www.cdc.gov/ vitalsigns/aian-diabetes/

18 Diné food Sovereignty: a report on the Navajo Naton food system and the case to rebuild a self-sufficient food system for the Diné people. Available: http://www.dinecollege.edu/institutes/DPI/Docs/ dpi-food-sovereignty-report.pdf

19 Navajo Nation Mortality Report, 2006 - 2009. Navajo Mortality Report New Mexico Portion, 2010 - 2013. Available: www.nec.navajo-nsn. gov

20 Navajo Nation Community Health and Outreach Program NDoH. Presentation by Mae-Gilene Begay, program director to Arizona Advisory Council on Indian healthcare, 2018.

21 King C, Goldman A, Gampa V, Smith C, et al. Strengthening the role of community health representatives in the Navajo nation. BMC Public Health 2017;17:348.

22 Behforouz HL, Farmer PE, Mukherjee JS. From directly observed therapy to accompagnateurs: enhancing AIDS treatment outcomes in Haiti and in Boston. Clin Infect Dis 2004;38:S429-36. 38.

23 Clarke V, Braun V, Terry G, et al. Thematic analysis. In: Liamputtong $\mathrm{P}$, ed. Handbook of research methods in health and social sciences. Springer: Singapore, 2019: 843-60.

24 SocioCultural Research Consultants Lwdc. Dedoose: Dedoose version 7.0.23, web application for managing, analyzing, and presenting qualitative and mixed method research data. Los Angeles, CA, 2016.

25 Centers for Disease Control and Prevention, U.S. Department of Health and Human Services. Community-Clinical Linkages for the Prevention and Control of Chronic Diseases: A Practitioner's Guide. Atlanta, GA, 2016.

26 Clinical-Community relationships evaluation roadmap. Available: https://www.ahrq.gov/prevention/resources/chronic-care/clinicalcommunity-relationships-eval-roadmap/index.html

27 Shah MK, Heisler M, Davis MM. Community health workers and the patient protection and Affordable care act: an opportunity for a research, advocacy, and policy agenda. J Health Care Poor Underserved 2014;25:17-24.

28 Otero-Sabogal R, Arretz D, Siebold S, et al. Physician-community health worker partnering to support diabetes self-management in primary care. Qual Prim Care 2010;18:363-72.

29 Bodenheimer T, Sinsky C. From triple to quadruple AIM: care of the patient requires care of the provider. The Annals of Family Medicine 2014;12:573-6.

30 Furukawa MF, King J, Patel V, Hsiao CJ, et al. Despite substantial progress in EHR adoption, health information exchange and patient engagement remain low in office settings. Health Aff 2014;33:1672-9. 